Research Article

\title{
Synthesis and Characterization of PVA-Assisted Metal Oxide Nanomaterials: Surface Area, Porosity, and Electrochemical Property Improvement
}

\author{
Buzuayehu Abebe $\mathbb{D}^{D}$, H. C. Ananda Murthy ${ }^{\mathbb{D}}$, and Enyew Amare Zereffa \\ Department of Applied Chemistry, School of Applied Natural Sciences, Adama Science and Technology University, P.O. Box: 1888, \\ Adama, Ethiopia
}

Correspondence should be addressed to Buzuayehu Abebe; buzea8@gmail.com and H. C. Ananda Murthy; anandkps350@gmail.com

Received 10 May 2020; Accepted 7 July 2020; Published 29 September 2020

Academic Editor: You Qiang

Copyright (c) 2020 Buzuayehu Abebe et al. This is an open access article distributed under the Creative Commons Attribution License, which permits unrestricted use, distribution, and reproduction in any medium, provided the original work is properly cited.

\begin{abstract}
The poly(vinyl alcohol)-assisted sol-gel-self-propagation route has been used for the synthesis of porous binary metal oxide nanocomposites (BMONCs) and ternary metal oxide nanocomposites (TMONCs). The effects of synthesis techniques, precursor's type, amount of PVA loading, and precursor's percentage were studied. The optical, chemical bonding, crystallinity, morphological, textural, and electrochemical properties of the synthesized materials were characterized by UV-vis-DRS/UV-vis, FT-IR, XRD, SEM/EDX and TEM/HRTEM/SAED, BET, and CV/EIS techniques, respectively. The porous nature of the materials was confirmed by SEM, BET, and SAED analytical techniques. Using XRD and TEM analysis, the approximate particle size of the materials was confirmed to be in the nanometer range $(\sim 7-70 \mathrm{~nm})$. The EDX and HRTEM analysis was confirming the presence of predictable composition and actuality of the composites, respectively. Moving from bare $\mathrm{ZnO}$ to ternary nanocomposites, the great morphological, surface area, and electrochemical property enhancement was confirmed. The charge transfer capability order was obtained to be $\mathrm{ZnO} / \mathrm{Fe}_{2} \mathrm{O}_{3} / \mathrm{Mn}_{2} \mathrm{O}_{3}>\mathrm{ZnO} / \mathrm{Fe}_{2} \mathrm{O}_{3}>\mathrm{ZnO} / \mathrm{Mn}_{2} \mathrm{O}_{3}>\mathrm{ZnO}$. The respective approximate electron transfer resistance value is $7,25,61$, and $65 \Omega$. Therefore, this work can improve the toxicity towards solvent used, surface area to volume ratio, and aggregation/agglomeration problem and also enhance the charge transfer capability due to the heterojunction.
\end{abstract}

\section{Introduction}

With the rapid development of industrial society, increasing environmental safety threats pose a major concern. Nowadays, metal oxides have been vigorously applied as an environmental waste management system [1]. Among several metal oxides, $\mathrm{ZnO}$ was reported to be less production cost, higher light absorption efficacy, and less toxic properties $[2,3]$. However, the dissolution in acidic solution, photocorrosion in alkaline solution under UV irradiation [4], fast electron-hole recombination during the photocatalytic reaction, and agglomeration are the obvious drawbacks of $\mathrm{ZnO}$ [5]. Among several efficient efforts applied to reduce the mentioned drawbacks, developing ZnO-based heterojunc- tion with different bandgap materials was obtained to be one of the preferences [6]. Depending on the magnetic property, stability, and high surface area to volume ratio property, iron and manganese oxides are reported to be promising materials [7-9].

Iron oxide utilizes efficient absorption of visible light during the formation of heterojunction with $\mathrm{ZnO}$ photocatalyst. As reported $[10,11]$, it can absorb approximately $20 \%$ of the solar spectrum. Depending on the applied temperature, among different crystalline polymorphs of $\mathrm{Fe}_{2} \mathrm{O}_{3}$ Viz. hematite $\left(\alpha-\mathrm{Fe}_{2} \mathrm{O}_{3}\right), \beta-\mathrm{Fe}_{2} \mathrm{O}_{3}$, maghemite $\left(\gamma-\mathrm{Fe}_{2} \mathrm{O}_{3}\right), \varepsilon-\mathrm{Fe}_{2} \mathrm{O}_{3}$, and $\zeta-\mathrm{Fe}_{2} \mathrm{O}_{3}$, the $\alpha-\mathrm{Fe}_{2} \mathrm{O}_{3}$ phase is the most stable and simple to be formed. The pathway for the formation of $\alpha-\mathrm{Fe}_{2} \mathrm{O}_{3}$ follows $\gamma-\mathrm{Fe}_{2} \mathrm{O}_{3} \rightarrow \mathcal{\varepsilon}-\mathrm{Fe}_{2} \mathrm{O}_{3} \rightarrow \alpha-\mathrm{Fe}_{2} \mathrm{O}_{3}$ [12]. Among different 
crystalline polymorphs of manganese oxides, the $\mathrm{Mn}_{2} \mathrm{O}_{3}$ is cheap and environmentally safe [13]. Both in the amorphous and crystalline state, $\alpha-\mathrm{Mn}_{2} \mathrm{O}_{3}$ uses a wide range of applications [14]. Manganese oxide also has low charge transfer resistance and multitudinous defect properties. In the nanosized range, $\alpha-\mathrm{Fe}_{2} \mathrm{O}_{3}$ and $\alpha-\mathrm{Mn}_{2} \mathrm{O}_{3}$ can enhance the adsorption capacity during heterojunction [15]. A significant surface area enhancement from single $\mathrm{ZnO}$ to ternary materials was also proven in the author's earlier work [16]. In addition to single $\mathrm{ZnO}$, this work compares additional two binary metal oxide composites with the ternary composite materials. Furthermore, the presence of good electrochemical improvement due to the formation of heterojunction was also proven.

Among several bottom-up synthesis approaches, depending on the price, time, easiness, and harmfulness, the sol-gel and coprecipitation techniques are feasible $[17,18]$. In advance, when being applied, toxic solvents, especially that are suggested under the severe human health risk phrase category of the Global Harmonized System (GHS) Hazard and Precautionary (H\&P), are cancer-causing and also have mutagenic properties [19]. As reported [20], the nontoxic solvents like water can give equivalent morphology and crystalline size. Furthermore, due to high surface energy and large surface area, metal oxides can aggregate/agglomerate easily. Among different techniques used to avoid the aggregation/agglomeration of the NPs, the polymer matrix as a capping agent is one of the preferences [21]. Due to several properties, poly(vinyl alcohol) (PVA) polymer has been reported to be efficient [22]. As reported [23, 24], $500^{\circ} \mathrm{C}$ is the optimum temperature to remove unwanted impurities including the PVA polymer after acting as a capping agent.

Therefore, considering all the mentioned aspects, this work was aimed at synthesizing single, BMONC, and TMONC materials using a simple and green sol-gel-self-propagation technique. The physical properties of the synthesized materials were characterized by $U V$-vis-diffuse reflectance spectroscopy (UV-vis-DRS), Fourier-transform infrared spectroscopy (FT$\mathrm{IR}), \mathrm{X}$-ray powder diffraction (XRD), scanning electron microscopy/energy-dispersive X-ray spectroscopy (SEM/EDX) and transmission electron microscopy/high-resolution transmission electron microscopy/selected area electron diffraction (TEM/HRTEM/SAED), Brunauer-Emmett-Teller (BET), and cyclic voltammetry/electrical impedance spectroscopy (CV/ EIS) analytical techniques. The techniques are confirming the presence of an enhanced surface area, porosity, and electrochemical improvement.

\section{Material and Methods}

2.1. Synthesis of Single, Binary, and Ternary Metal Oxides. The common analytical grade reagents and characterization technique details were present as supplementary materials (S). The single, binary, and ternary nanomaterials were synthesized following the sol-gel-self-propagation process. In detail, the PVA polymer was dissolved in distilled water while stirring on a magnetic stirrer at $\sim 115^{\circ} \mathrm{C}$ for about 15 minutes [25]. After cooling at room temperature, the $\mathrm{Zn}, \mathrm{Mn}$, and $\mathrm{Fe}$ salt precursors (18:5:5 molar ratio, respectively) were added

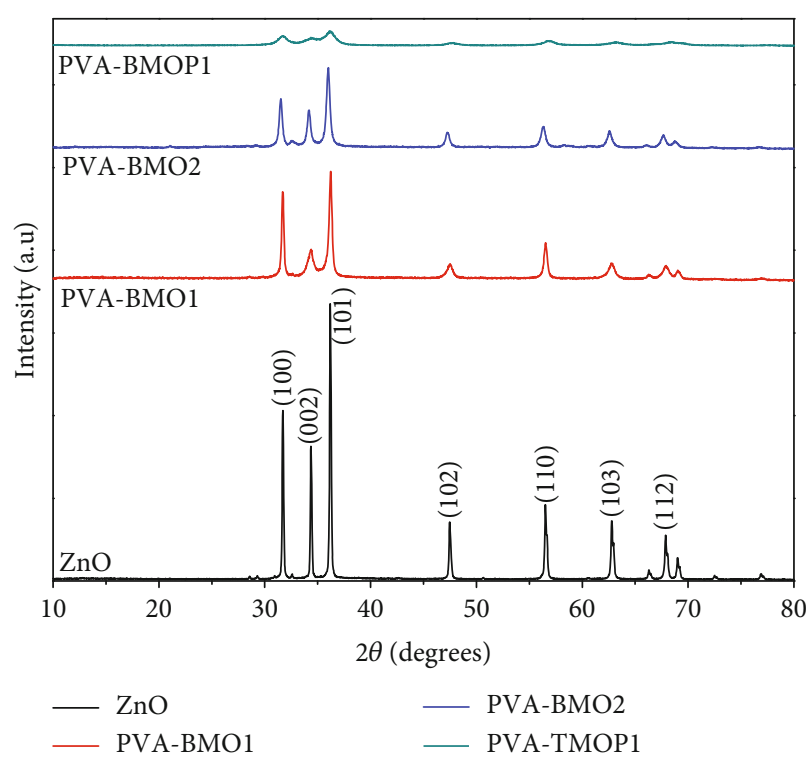

Figure 1: XRD patterns of ZnO, PVA-BMO1, PVA-BMO2, and PVA-TMOP1 nanomaterials.

to form a $0.01 \mathrm{~mol} \mathrm{~L}^{-1}$ aqueous metal salt solution. The sol formed was aged for two days to form a gel and dried in an oven at $110^{\circ} \mathrm{C}$. At the final drying process, the unintentional self-propagation process took place and a highly porous product was formed. The dried gel was grounded and calcined at $500^{\circ} \mathrm{C}$ for 3 hours. The detailed procedure is also reported on the author's earlier works $[16,26]$ and shown in Figure S1. The same protocol was used for the synthesis of binary metal oxides by mixing two different metal salt precursors.

To synthesize single $\left(\mathrm{ZnO}, \mathrm{Fe}_{2} \mathrm{O}_{3}\right.$, and $\left.\mathrm{Mn}_{2} \mathrm{O}_{3}\right)$, binary, and ternary metal oxides without PVA, except the first procedure used to dissolve the polymer, the same protocols were followed. However, due to the absence of the polymer, no self-propagation process occurred at the final drying period. Single $\mathrm{ZnO}$ was synthesized using a zinc nitrate salt precursor. The synthesized PVA-assisted BMONCs (PVA-BMONCs) were coded as PVA-BMO1 and PVA$\mathrm{BMO} 2$ for $\mathrm{Zn}-\mathrm{Fe}$ and $\mathrm{Zn}-\mathrm{Mn}$ oxides, respectively. The respective BMONCs synthesized without PVA were coded as BMO1 and BMO2 (see Table S1 and S2). The synthesized TMONCs without PVA using the sol-gel (M1) and coprecipitation (M2) techniques were coded as TMOM1 and TMOM2, respectively, whereas the PVA-assisted TMONCs (PVA-TMONCs) using zinc nitrate (P1) and zinc acetate (P2) precursors were coded as PVA-TMOP1 and PVATMOP2, respectively (see Table S3).

\section{Results and Discussion}

3.1. XRD Analysis. The XRD diffraction pattern of the synthesized nanomaterials that confirms the crystallinity and composition of single, BMONC, and TMONC materials are shown in Figure 1. All the diffraction angles with its 


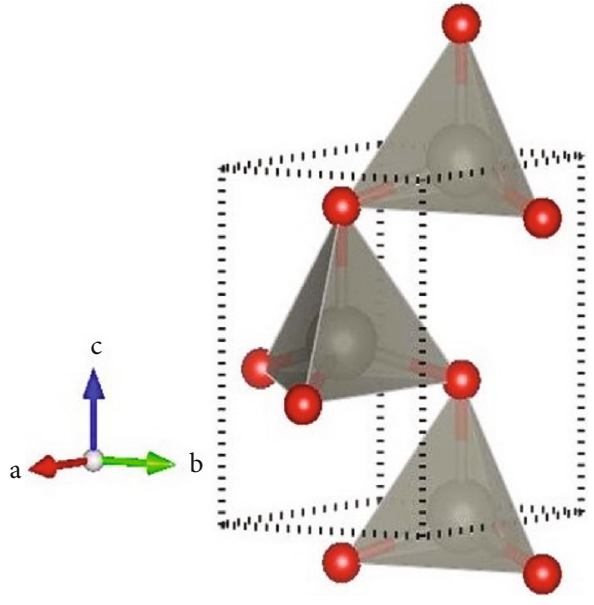

(a)

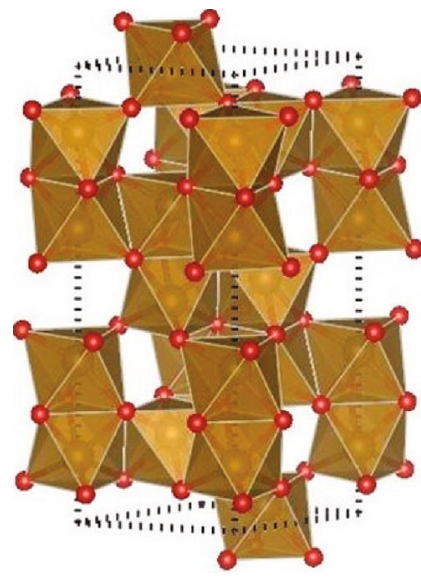

(b)

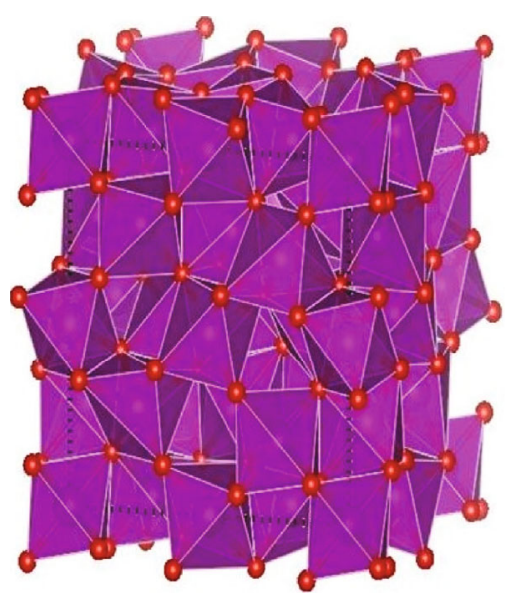

(c)

Figure 2: The polyhedral style crystal structures of (a) $\mathrm{ZnO}$, (b) $\mathrm{Fe}_{2} \mathrm{O}_{3}$, and (c) $\mathrm{Mn}_{2} \mathrm{O}_{3}$ (red is for O atoms).

corresponding crystal planes are corresponding to $\mathrm{ZnO}$ (ICSD: 00-036-1451, P63mc (\#186-1) space group) (Figure 1). For single $\mathrm{Fe}_{2} \mathrm{O}_{3}$, the diffraction angles with its corresponding crystal planes are consistent with hematite, $\alpha-\mathrm{Fe}_{2} \mathrm{O}_{3}$ (ICSD: 00-033-0664, R-3c (\#167-1) space group) (see Figure S2(a)). Furthermore, for single $\mathrm{Mn}_{2} \mathrm{O}_{3}$, the diffraction angles with its corresponding crystal planes are consistent with $\alpha-\mathrm{Mn}_{2} \mathrm{O}_{3}$ (ICSD: 24342, PBCA space group) (Figure $\mathrm{S} 2(\mathrm{~b})$ ). The structure of stable $\mathrm{ZnO}, \alpha$ $\mathrm{Mn}_{2} \mathrm{O}_{3}$, and $\alpha-\mathrm{Fe}_{2} \mathrm{O}_{3}$ that was developed using the VESTA $3 \mathrm{D}$ visualization program software depending on the AMCSD search result is given in Figures 2(a)-2(c), respectively.

The particle size optimizations for BMONCs with and without PVA are given in Figure S2(a) and (b). For the TMONCs, the particle size optimizations using different methods and salt precursors are given in Figure S2c. Further particle size optimizations using different amounts of PVA and precursor percentages were also present in [16]. For the BMONCs and TMONCs, except for peak intensity reduction and width widening, no diffraction peaks for $\mathrm{Fe}_{2} \mathrm{O}_{3}$ and $\mathrm{Mn}_{2} \mathrm{O}_{3}$ were found. Most probably, this is due to the small percentage of iron and manganese oxides. The nonshifting of peak position may also indicate the absence of any structural distortion on the $\mathrm{ZnO}$ crystal lattice attributed to $\mathrm{Fe}^{3+}$ or $\mathrm{Mn}^{3+}$ ion inclusion [27]. This means $\mathrm{Fe}_{2} \mathrm{O}_{3}$ and $\mathrm{Mn}_{2} \mathrm{O}_{3}$ are present as a separate phase through forming only a local heterojunction [27]. Loading a higher percentage of iron or/and manganese precursors above the optima may also degrade the structure of $\mathrm{ZnO}$ [28]. Furthermore, due to the presence of ionic radius differences between zinc and iron or/and manganese oxides, instead of doping, the formation of heterojunction was recommended [29].

A reasonable surface area enhancement for PVABMONCs and PVA-TMONCs was observed compared to single $\mathrm{ZnO}, \mathrm{BMONCs}$, and TMONCs (Table S1-3). In advance, compared to the PVA-BMONCs, the PVATMONCs are showing enhanced surface area results. The
$\mathrm{XRD}$ data and the respective size of the particles were calculated using Debye-Scherrer's formula:

$$
D=\frac{K \lambda}{\beta \cos (\theta)}
$$

where $\lambda$ is the wavelength of $\mathrm{X}$-ray radiation (for $\mathrm{Cu}$ $0.15418 \mathrm{~nm}), K$ is constant, $\beta$ is the full width at half maximum (FWHM), and $\theta$ is the diffracting angle.

3.2. UV-vis-DRS and UV-vis Studies. The optical properties of the nanopowder and its aqueous solution were characterized by UV-vis-DRS and UV-vis techniques, respectively. The percent reflectance versus wavelength along with the Kubelka-Munk (K-M) plots of $\mathrm{ZnO}$ powder is given in Figure 3. $\mathrm{ZnO}$ and the optimized PVA-BMO1, PVABMO2, and PVA-TMOP1 nanocomposites are showing a characteristic absorption edge near to $380 \mathrm{~nm}$ (Figure 3(a)). Compared to the other, pure $\mathrm{ZnO}$ is showing high reflectance in the visible region. In fact, due to its wide bandgap energies $(3.17 \mathrm{eV})$, it only responds to the UV region. However, for the binary and ternary composites, the reflectance in the visible region is reduced. Compared to $\mathrm{ZnO}$, PVA-TMO1 and PVA-TMOP1 have low reflectance $(\sim 45 \%)$. This enhanced optical activity for the composites is due to the increased porosity/surface imperfection [30].

In the parabolic band structure, the bandgap energy, $E_{g}$, and the absorption coefficient of a direct bandgap semiconductor $\alpha$ are related through

$$
(\alpha \hbar v)^{1 / n}=A\left(\hbar v-E_{g}\right)
$$

where $\hbar$ is Planck's constant, $v$ is the photon's frequency, and $A$ is the slope in the linear region. Taking the $\mathrm{K}-\mathrm{M}$ scattering coefficient $S$ as a constant concerning the wavelength, and 


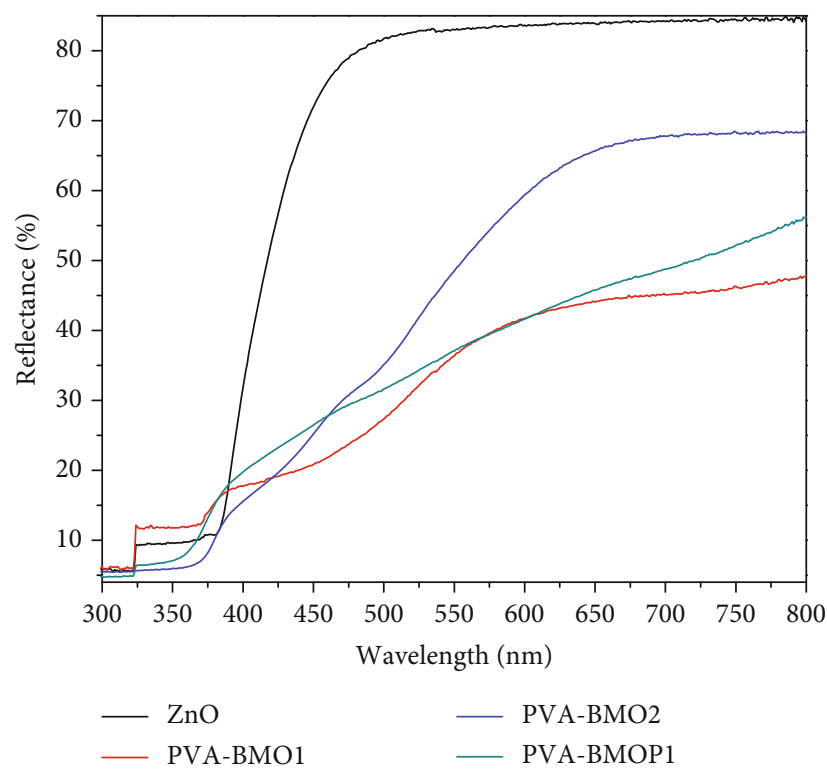

(a)
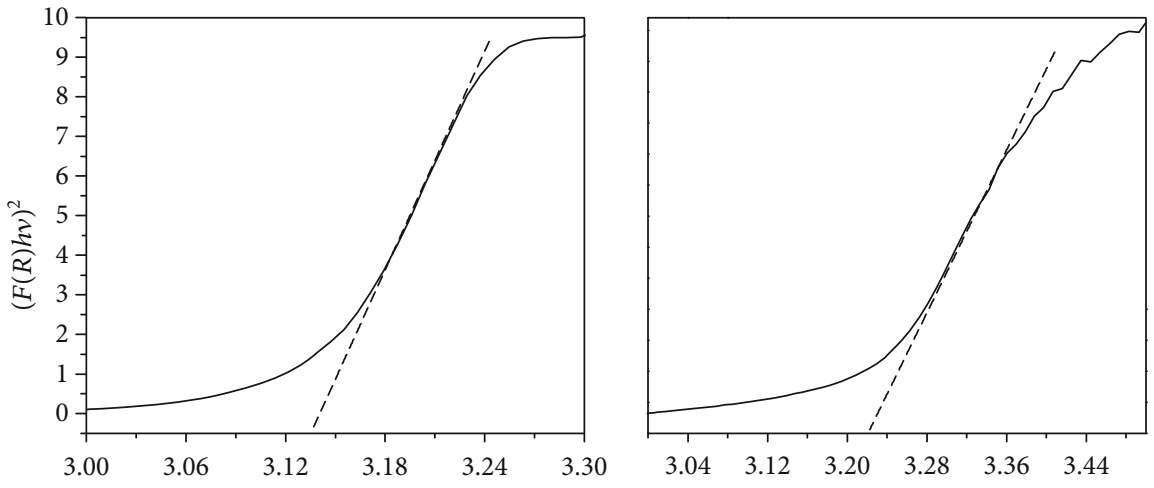

$-\mathrm{ZnO}$

— PVA-BMO1
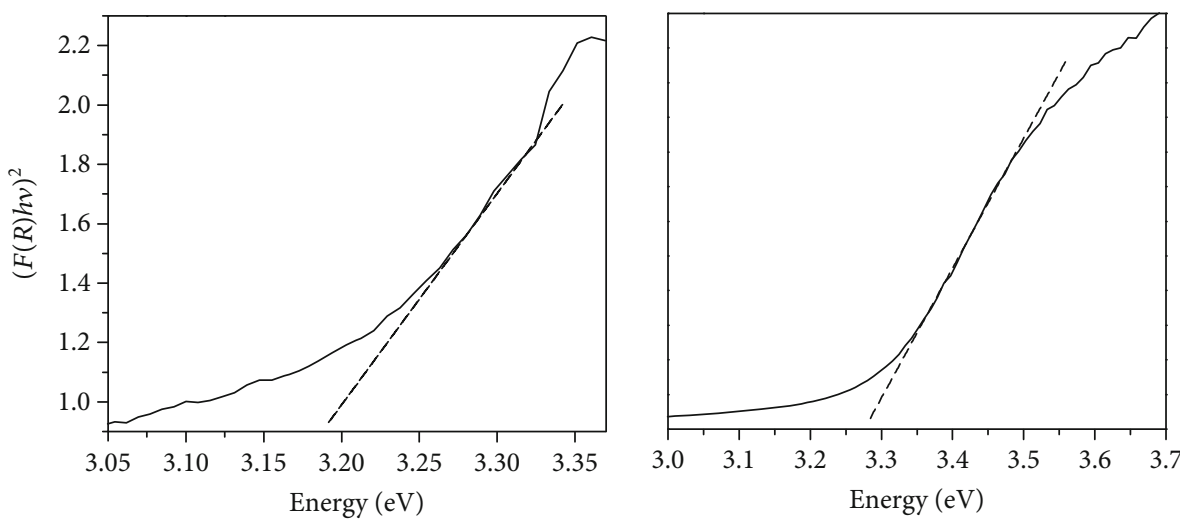

PVA-BMO2

— PVA-TMOP1

(b)

Figure 3: Continued. 

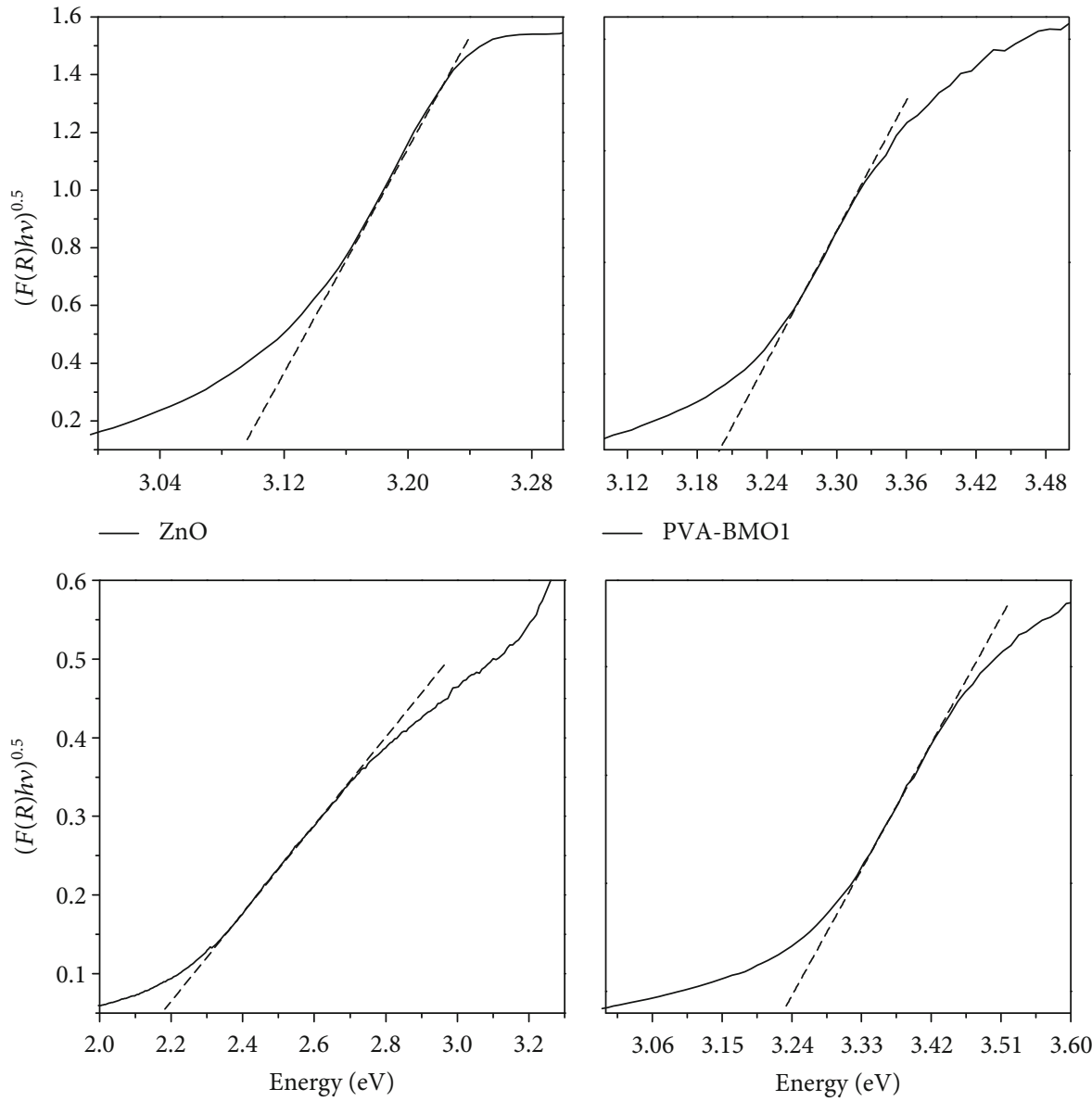

- PVA-BMO2

(c)

Figure 3: (a) DRS plots of ZnO, PVA-BMO1, PVA-BMO2, and PVA-TMOP1; the respective direct (b); and indirect (c) Kubelka-Munk plots.

using the remission function in Equation (2), the following expression was derived:

$$
(F(R) \hbar v)^{2}=A\left(\hbar v-E_{g}\right)
$$

where $F(R)$ is equal to $K / S$, the molar absorption coefficient $K$ is equal to $(1-R)^{2}$, the scattering factor $S$ is equal to $2 R$, and $R$ is the reflectance of the materials and is defined as $\% R / 100$.

As shown in Figures 3(b) and 3(c), the direct and indirect bandgap energies of the $\mathrm{ZnO}, \mathrm{PVA}-\mathrm{BMO} 1, \mathrm{PVA}-\mathrm{BMO} 2$, and PVA-TMOP1 materials were measured by the extrapolation of the linear portion of the direct $\mathrm{K}-\mathrm{M}$ function plots. The result shows the presence of nonnoticeable bandgap change. As shown in Figure S3(a) and (b), the nonobvious bandgap change was also further confirmed on UV-vis analysis conducted for optimized PVA-TMOP1 nanocomposites during the PVA amount and precursor percentage particle size optimization [16]. This may be due to the nonincorporation of either $\mathrm{Fe}^{0} / \mathrm{Mn}^{0}$ or $\mathrm{Fe}^{3+} / \mathrm{Mn}^{3+}$ ions in the $\mathrm{ZnO}$ crystalline lattice as confirmed on the XRD pattern [31]. The formation of this type of couple/heterojunction between metal oxides can enhance the charge transfer property. Furthermore, compared to single $\mathrm{ZnO}$, the observed slight bandgap tuning (blue shift) for the composite could be attributed to the confinement effects [32].

3.3. FT-IR Analysis. The FTIR technique that gives the chemical bonding information of single, binary, and ternary nanomaterials is shown in Figure 4. The calcined (C) and uncalcined (U) PVA-BMONCs and PVA-TMONCs are also given in Figure S4(a)-(c). The common broad absorption band around $\sim 3600$ and $\sim 1650 \mathrm{~cm}^{-1}$ is due to the stretching vibration of chemisorbed hydroxyl groups and physisorbed water molecules. Due to interatomic vibration, semiconductor metal oxides exhibit a strong absorption band below $1000 \mathrm{~cm}^{-1}$. Due to two transverse optical stretching modes, the FT-IR spectrum of $\mathrm{ZnO}$ shows a characteristic absorption band in the range of $400-550 \mathrm{~cm}^{-1}$. These absorption peaks correlated with the TO-phonon and the LO-phonon frequency [33]. The shape, number, and wavenumber position of the bands are dependent on the chemical composition, morphology, and crystal structure of the materials. This means if the morphology of the materials changed from spherical (zero) dimension to one, two, or three-dimensional morphology, the 


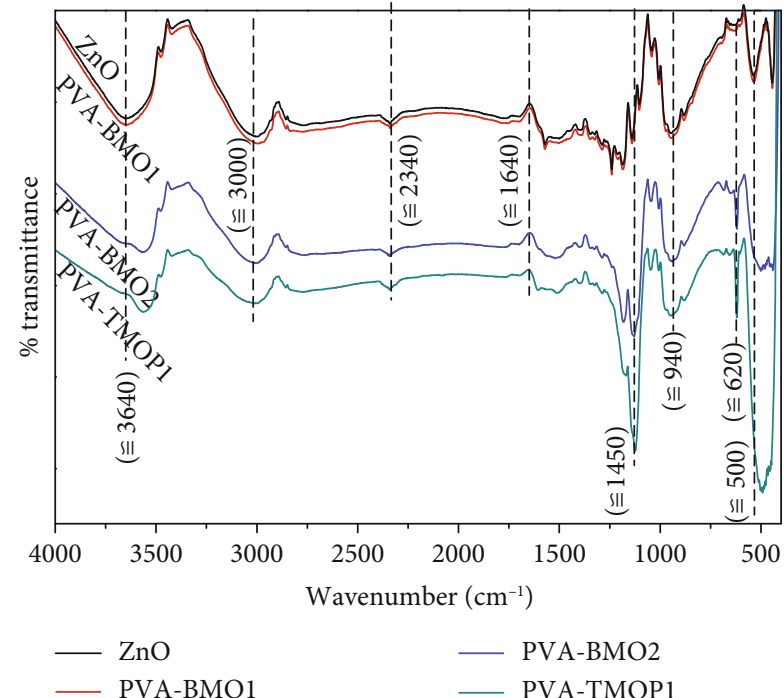

Figure 4: FT-IR spectra of ZnO, PVA-BMO1, PVA-BMO2, and PVA-TMOP1 nanomaterials.

broadness and splitting were also found to increase [34, 35]. For the crystalline $\mathrm{ZnO}$, within the range of $400-550 \mathrm{~cm}^{-1}$, the two absorption peaks were observed. For PVA composites, only one peak was observed.

Comparing the spectra of $\mathrm{ZnO}$ and calcined PVA composites with uncalcined PVA composites, one distinctive peak appeared around $\sim 1400 \mathrm{~cm}^{-1}$ is due to the $-\mathrm{CH}_{2}$ bending vibration of PVA [36, 37]. Furthermore, the wavenumber shift and intensity difference were also observed. As reported [38], the source for the other peaks is expected to be from intermediate impurities created during synthesis. The absorption peaks appeared at $\sim 1640$ and $\sim 1400 \mathrm{~cm}^{-1}$ are most probably either due to $\mathrm{C}=\mathrm{C}$ and $\mathrm{C}-\mathrm{C}$ stretching, respectively, or from the symmetric and asymmetric stretching vibration of the $\mathrm{C}=\mathrm{O} / \mathrm{C}-\mathrm{O}$ group. The absorption peaks appeared at $\sim 3000 \mathrm{~cm}^{-1}$ are due to the symmetric and asymmetric stretching vibration of $\mathrm{CH}_{2}[39,40]$. The absorption bands appeared at $\sim 1300$ and $\sim 940 \mathrm{~cm}^{-1}$ were ascribed to symmetric $\mathrm{C}-\mathrm{H}$ and bending vibration of the $-\mathrm{CH}_{3}$ group, respectively. The peak at $\sim 1100 \mathrm{~cm}^{-1}$ is identified as CO $(\mathrm{H})$ and $\mathrm{CO}(\mathrm{C})$ vibrations. Comparing the broad absorption band of $\mathrm{ZnO}$ with PVA composites, shifting of PVA composite peaks towards lower wavenumber was observed. The observed shift for both calcined and uncalcined PVA composites indicates surface passivation influences of PVA during synthesis. Indirectly, this indicates the presence of hydrogen bonds between the PVA (O-H group) and metal oxide surface [41].

3.4. BET Analysis. For an efficient environmental west management system, the surface-active sites and optimized pore volume of the synthesized materials are important [42]. The BET plots of single, binary, and ternary metal oxides are given in Figure S5-8. Furthermore, the combined BET plots of ZnO, PVA-BMO1, PVA-BMO2, and PVATMOP1 are also given in Figure 5. As seen in BET plots, the nanomaterials have a resemblance to the cylindrical pore shape model. The BET data is treated according to a $\mathrm{BET}$ adsorption isotherm linear equation:

$$
\frac{P / P_{o}}{n\left(1-P / P_{o}\right)}=\frac{C-1}{\left(n_{m} C\right)}\left(\frac{P}{P_{o}}\right)+\frac{1}{n_{m} C}
$$

where $P_{o}$ is the saturated vapor pressure of $\mathrm{N}_{2}$ gas in $\mathrm{Pa} ; P$ is the partial vapor pressure of $\mathrm{N}_{2}$ gas in $\mathrm{Pa} ; n$ is the volume of $\mathrm{N}_{2}$ gas adsorbed at standard temperature and pressure (STP) in $\mathrm{mL} ; n_{m}$ is the BET monolayer capacity; $C$ is the dimensionless constant related to the enthalpy of $\mathrm{N}_{2}$ gas adsorption on the single, BMONC, and TMONC adsorbent.

According to the BET theory, the value of $C$ has a close relationship with the shape of the isotherms. For defining the BET monolayer capacity of the nanocomposites, the value of $C$ should be $\geq 80$ [43]. As seen from Table S4-6, the values of $\mathrm{C}$ for $\mathrm{ZnO}, \mathrm{Mn}_{2} \mathrm{O}_{3}, \mathrm{BMO}, \mathrm{BMO}$, and PVA$\mathrm{BMO} 1$ are $<80$ and the $C$ values for the other are $>80$. For PVA-BMO1, PVA-TMOM1, and PVA-TMOP1, since their slope is 0 , it is not possible to define the value of $C$. The specific surface area in $\mathrm{m}^{2} \cdot \mathrm{g}^{-1}$ is calculated as

$$
a_{s}=\frac{n_{m} L \sigma_{\mathrm{m}}}{m \times 22400},
$$

where $a_{s}$ is the BET-specific surface area of the single, BMONCs, and TMONCs of mass $m$ in grams, $L$ is the Avogadro constant $\left(6.022 \times 10^{23} \mathrm{~mol}^{-1}\right), \sigma_{m}$ is the molecular adsorptive cross-sectional area occupied by $\mathrm{N}_{2}$ gas molecule in the complete monolayer (equal to $0.162 \mathrm{~nm}^{2}$ for $\mathrm{N}_{2}$ gas), and the 22400 is the volume occupied by 1 mole of $\mathrm{N}_{2}$ gas at $\mathrm{STP}$, in $\mathrm{mL}$.

Among six types of adsorption isotherms that are characteristic of porous materials (types I-VI) [44], the appearances of the synthesized materials look like a typical IV isotherm with an $\mathrm{H} 3$ hysteresis loop. High adsorption capacities and sharp inflection at a high relative pressure $(P / P 0>0.8)$ signifying the coexistence of mesopore and macropore size distribution [45]. The sharp capillary condensation step between 0.40 and 0.85 for PVA-TMOP2 reflects the presence of a narrow pore size distribution.

The relatively large surface area and narrow mesoporous channels provide enough space and facilitate the rapid adsorption of molecules. The pore size distributions of the synthesized materials were expressed in terms of the Barrett-Joyner-Halenda (BJH) curve. According to the IUPAC classification, materials that have a pore size diameter of $<2.0 \mathrm{~nm}$ is categorized as microporous, in the range between 2.0 and $50.0 \mathrm{~nm}$ as mesoporous, and $>50.0 \mathrm{~nm}$ as macroporous [43, 46, 47]. As seen in Table S4-6, for all single, BMONCs, and TMONCs, the average pore size distribution is in the range of $9-43 \mathrm{~nm}$. This indicates the materials have mesoporous pore size distribution properties. Except for $\mathrm{Fe}_{2} \mathrm{O}_{3}$, the sizes of mesopores in the architecture of all materials look uniform; the difference is only in intensity. The appearance of the peaks $>60 \mathrm{~nm}$ indicates the presence of some macroporous size distribution (see Figure S5-8 inset) [45]. Therefore, the materials have mesomacropore size distribution properties, yet the mesoporous 


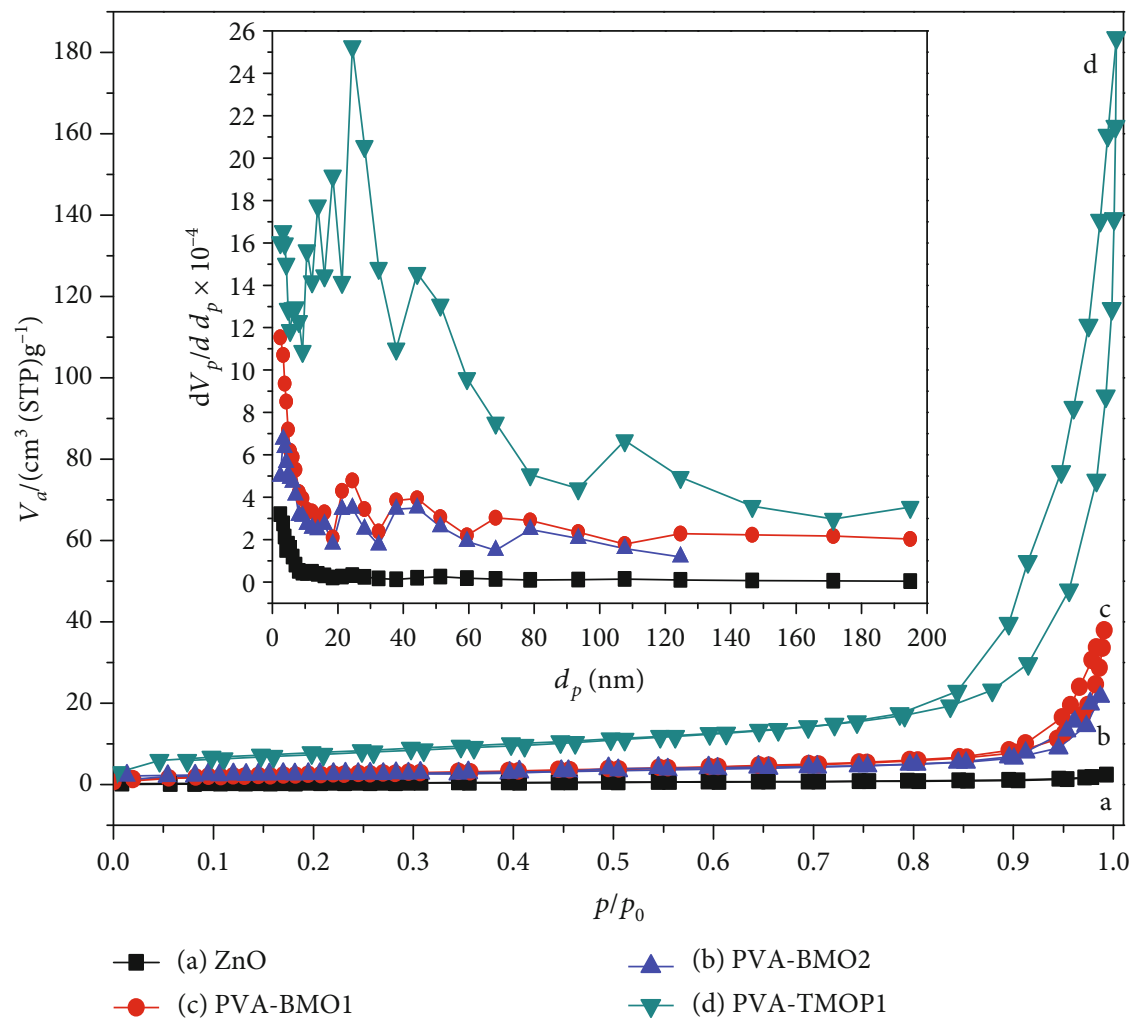

Figure 5: BET plots of ZnO (a), PVA-BMO1 (c), PVA-BMO2 (b), and PVA-TMOP1 (d) (inset: the respective BJH pore size distributions).

type is dominant. The pore size curve shows a relatively wide distribution for PVA-TMOP1, which is consistent with the SEM result. Compared to single, BMONCs, and TMONCs, the PVA-TMOP1 is showing enhanced surface area and optimized pore volume.

3.5. SEM-EDX Analysis. For morphological and microstructure properties of synthesized materials, the SEM analysis was studied. The SEM images of $\mathrm{ZnO}, \mathrm{BMO} 1, \mathrm{BMO} 2$, TMOM1, TMOM2, and PVA-TMOP2 nanomaterials are given in Figure S9(a)-(f), respectively, whereas the SEM images and the respective EDX spectra of PVA-BMO1, PVA-BMO2, and PVA-TMOP1 are given in Figures 6(a)6(c). The morphology of BMONCs and TMONCs shows greater crystallinity compared to PVA-BMONCs and PVATMONCs. This is consistent with the XRD pattern result of the materials. Furthermore, as seen on SEM images, BMONCs and other TMONCs have high agglomeration/ aggregation properties than PVA-BMONCs and PVATMONCs.

Also, as confirmed on the $\mathrm{N}_{2}$ adsorption study, the porosity of PVA-BMO1 and PVA-TMOP1 is more visible than the others (see Figures 6(a) and 6(b)). To confirm the presence of $\mathrm{Zn}, \mathrm{Mn}, \mathrm{Fe}$, and $\mathrm{O}$ compositions, which are not detected on the SEM images and XRD pattern, the EDX analysis was conducted. The result showed the presence of the expected $\mathrm{Zn}, \mathrm{Mn}, \mathrm{Fe}$, and $\mathrm{O}$ elements. However, $\mathrm{S}$ in PVATMOP1, $\mathrm{Cl}$ and $\mathrm{N}$ in PVA-BMO1, and $\mathrm{C}$ and $\mathrm{S}$ in PVABMO2 present as impurities. The source for these impurities is most probably from the standard used during analysis $[48,49]$.
3.6. TEM Analysis. The TEM morphology of the optimized PVA-TMOP1, PVA-BMO1, and PVA-BMO2 NCs is shown in Figures 7-9, respectively. As seen in Figure 7(a) of the PVA-TMOP1 image, agglomerates with a size ranging from 7 to $30 \mathrm{~nm}$ were observed. The particle size diameter for PVA-BMO1 and PVA-BMO2 composites is in the range of 20-70 and 30-60 $\mathrm{nm}$, respectively. This is in agreement with the obtained approximate particle size from each XRD pattern. The TEM analysis also confirmed the porous nature of the composite, which is in good agreement with BET and SEM analysis. Between the agglomerates of PVA-TMOP1, PVA-BMO1, and PVA-BMO2 NCs, the oriented attachment that usually occurs between metal oxides was also detected. During oriented attachment, the presence of fission as a driving force and the particles were found to share a common crystallographic orientation $[50,51]$.

The output from the lattice fringe analysis is given in Figures $7(\mathrm{~d})$ and $7(\mathrm{e})$ for PVA-TMOP1 and Figures $8(\mathrm{~d})$ and $9(\mathrm{~d})$ for PVA-BMO1 and PVA-BMO2, respectively. Figure $7(\mathrm{c})$ shows the magnified HRTEM image of PVATMOP1. From characteristic lattice fringes that appeared on the HRTEM image, the $d$-spacing value for (002) plane of $\mathrm{ZnO}$ was found to be $0.28 \mathrm{~nm}$. The $d$-spacing value of $0.34 \mathrm{~nm}$ is matching to (221) plane of $\alpha-\mathrm{Mn}_{2} \mathrm{O}_{3}[52,53]$. Yet, the lattice fringes for $\alpha-\mathrm{Fe}_{2} \mathrm{O}_{3}$ were not found on the HRTEM image which is possibly due to the highly dispersed states of it. The lattice fringes of $\alpha-\mathrm{Fe}_{2} \mathrm{O}_{3}$ were also not detected on the PVA-BMO1. Furthermore, as seen in Figure $9(\mathrm{~d})$, the $d$-spacing value of $\sim 0.343 \mathrm{~nm}$ is corresponding to (221) atomic plane of $\alpha-\mathrm{Mn}_{2} \mathrm{O}_{3}$. In advance, unlike 

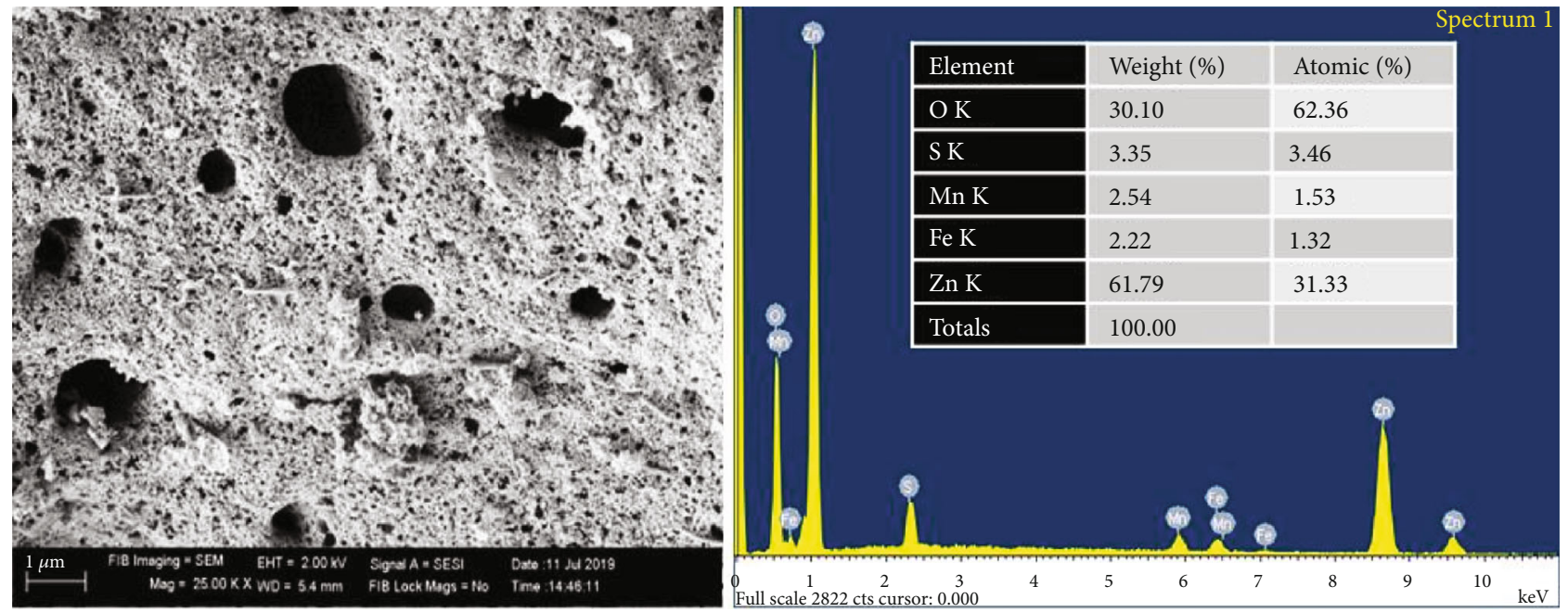

(a)
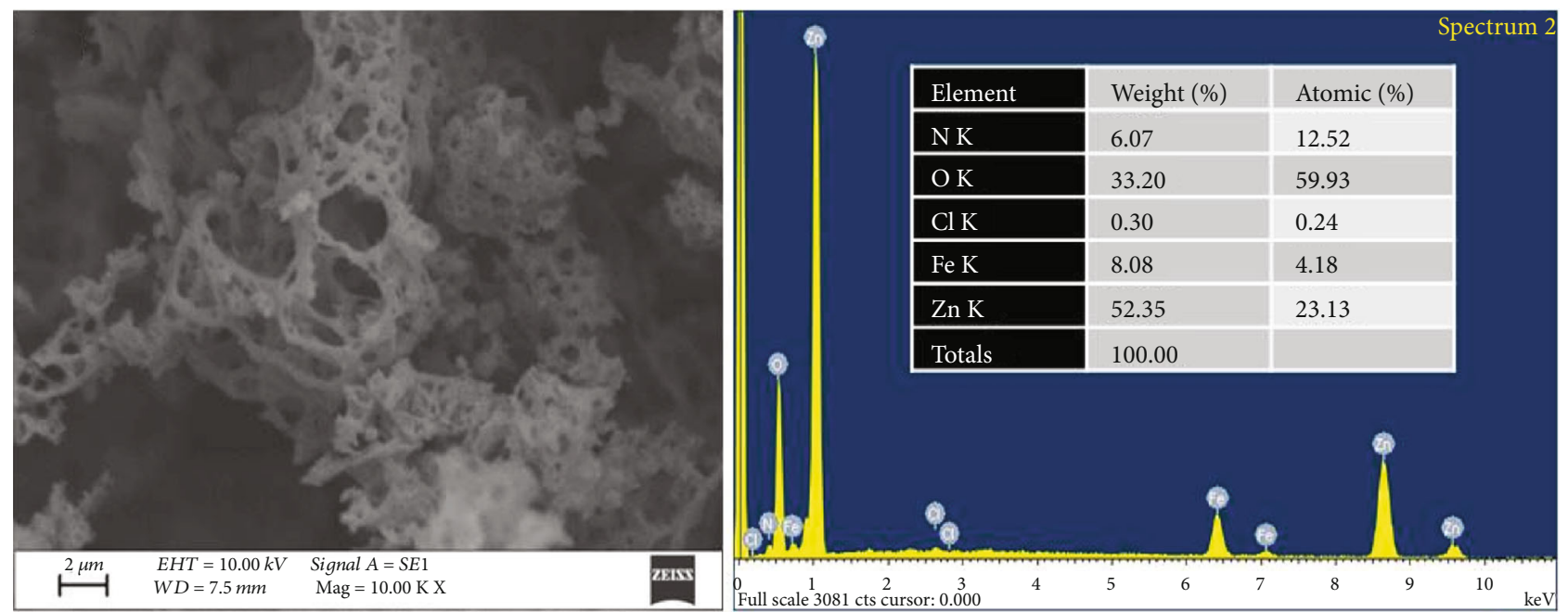

(b)
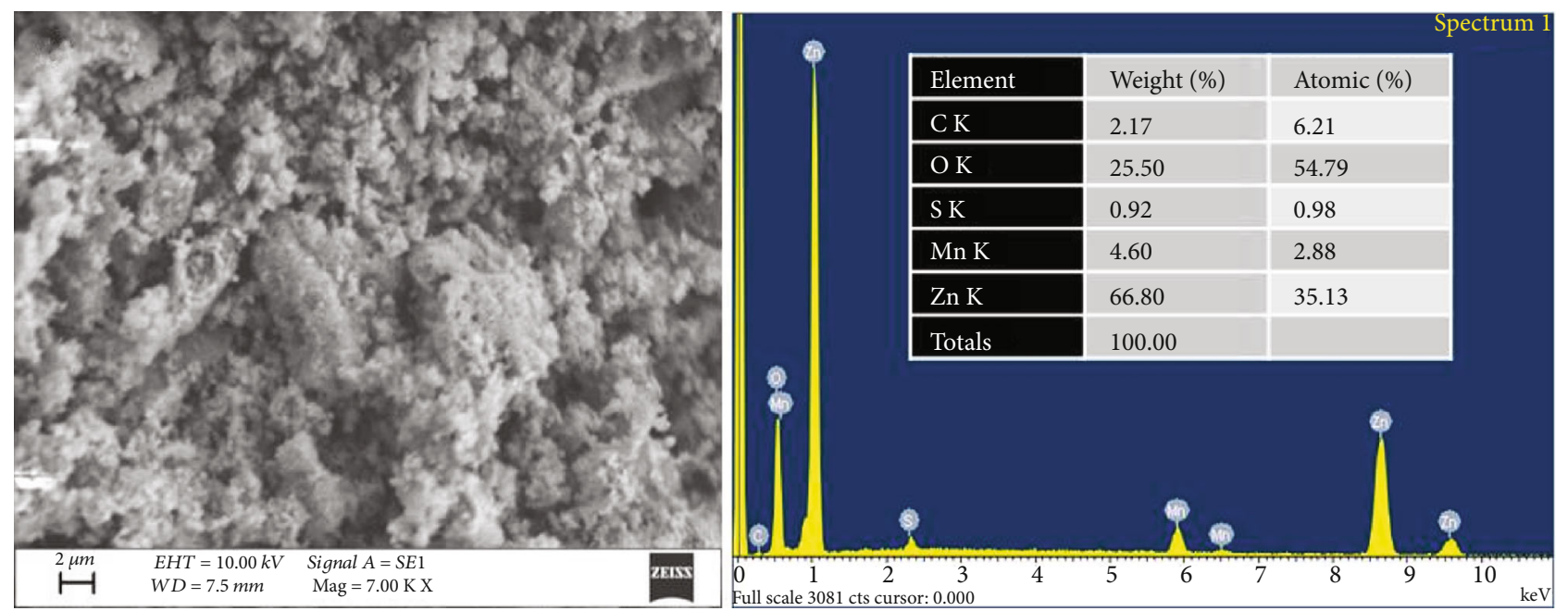

(c)

FIgURE 6: SEM images and the respective EDX spectra of (a) PVA-TMOP1, (b) PVA-BMO1, and (c) PVA-BMO2 nanocomposites.

that of PVA-BMO1 and PVA-TMOP1 (Figures $7(\mathrm{a})$ and $8(\mathrm{a}))$, the presence of two different morphologies in
Figure 9(a) clearly shows the existence of two different components. 


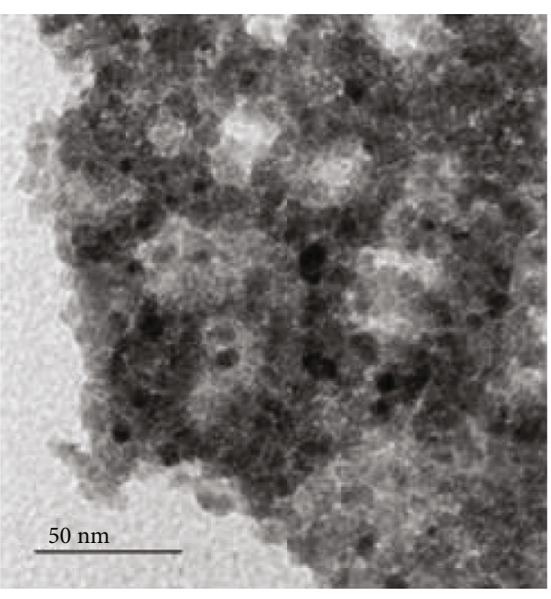

(a)

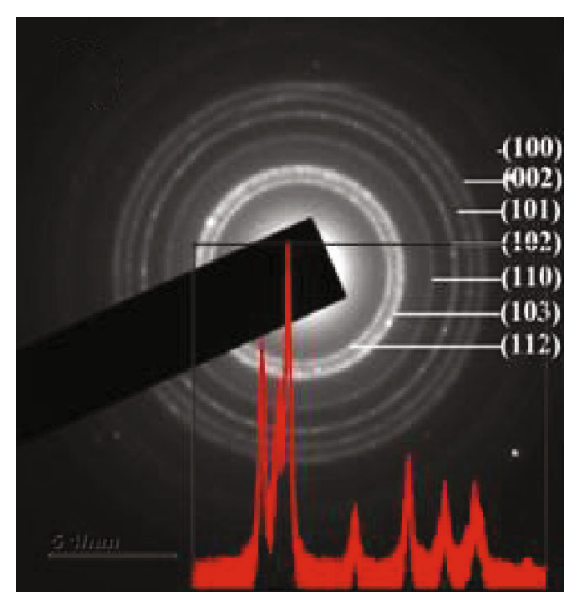

(b)

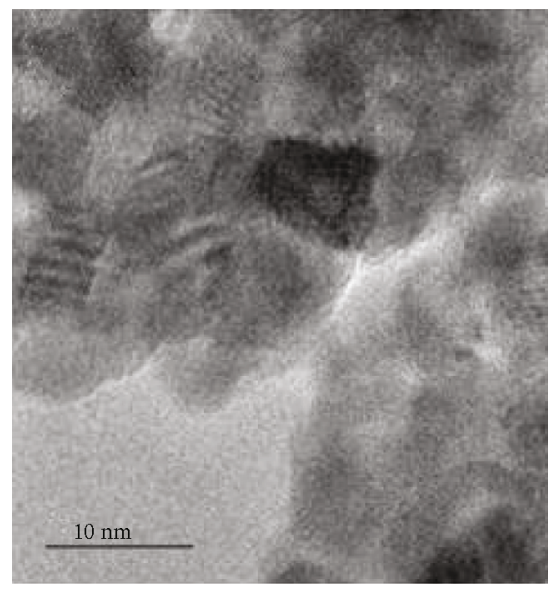

(c)

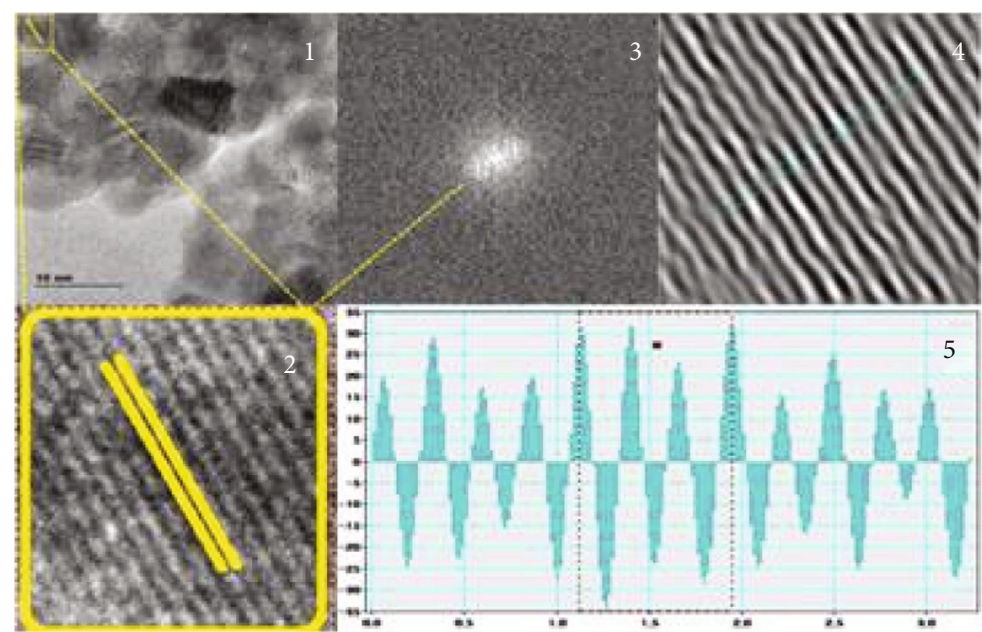

(d)
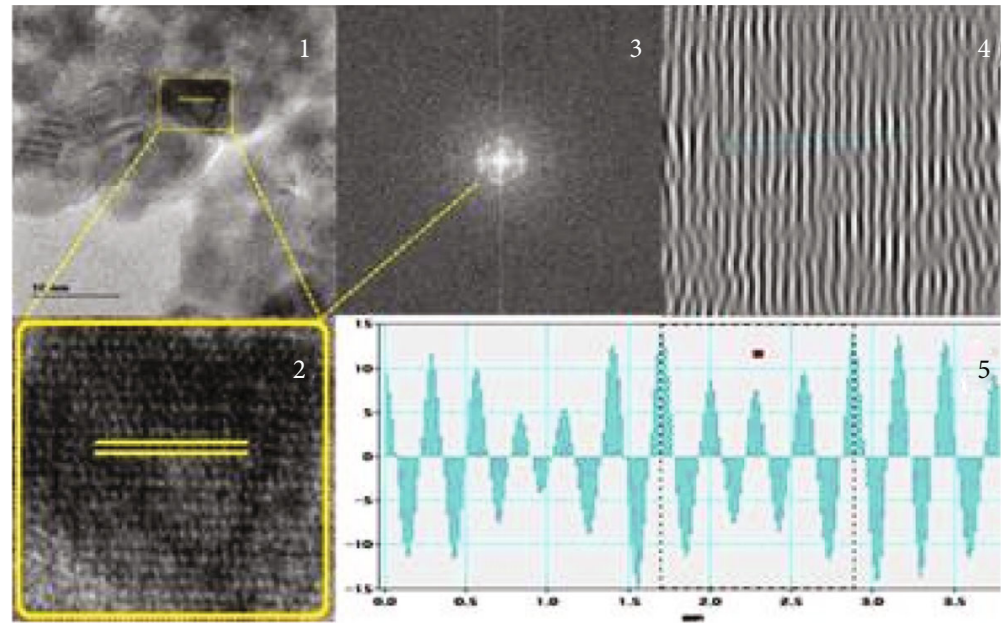

(e)

Figure 7: (a) TEM image of PVA-TMOP1; (b) SAED ring (inset: XRD pattern of PVA-TMOP1); (c) HRTEM image; images (2), (3), (4), and (5) for $\mathrm{ZnO}(\mathrm{d})$ and $\mathrm{Mn}_{2} \mathrm{O}_{3}$ (e) are the magnified, FFT, IFFT, and profile of IFFT image of (1), respectively.

The occurrence of stacking faults on the surface of the NPs suggests the semicrystalline nature of the nanocomposites (see the IFFT (4) images of Figures 7-9). The selected area electron diffraction (SAED) rings of PVA-TMOP1,
PVA-BMO1, and PVA-BMO2 are given in Figures 7(b), $8(\mathrm{c})$, and $9(\mathrm{c})$, respectively. The obtained approximate interplanar spacing of the diffraction rings from SAED ring matches with XRD pattern of the respective composites. 


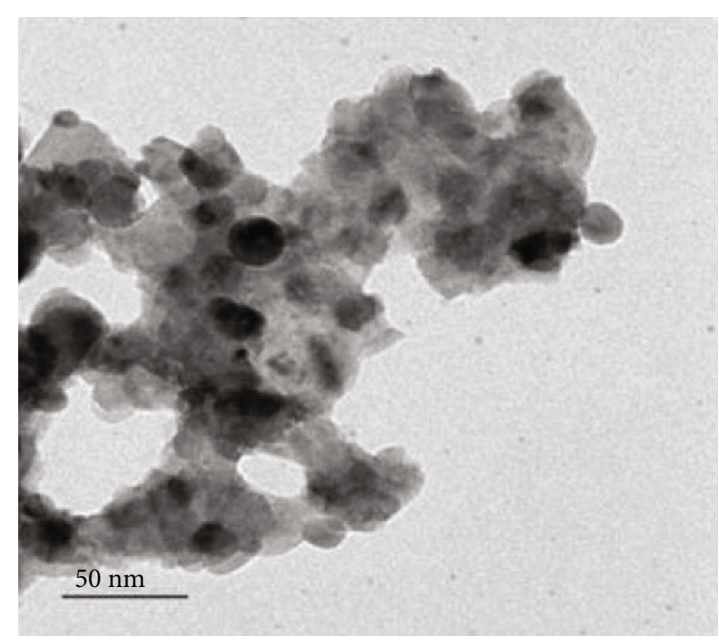

(a)

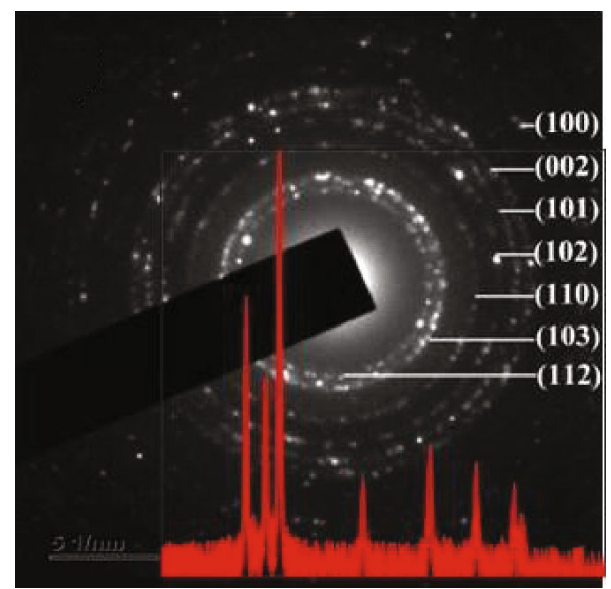

(c)

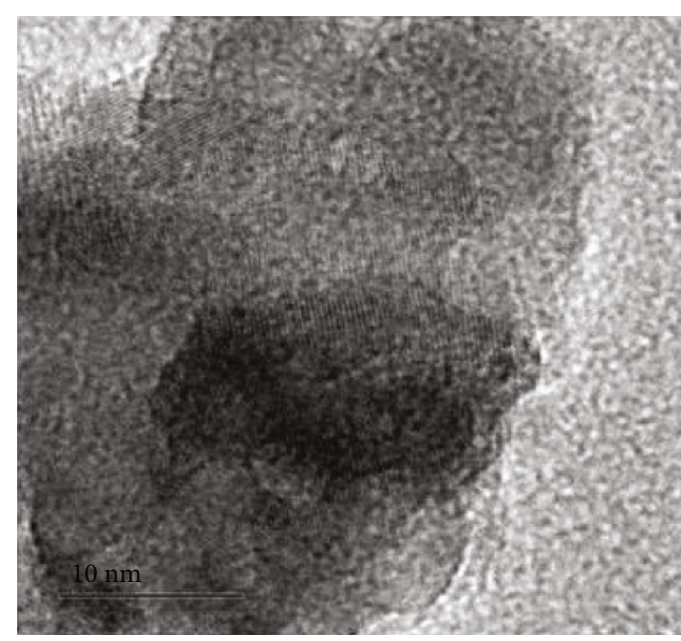

(b)

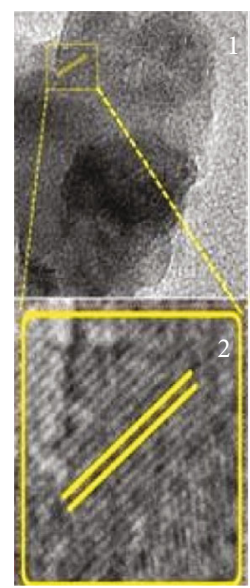

$(\mathrm{d})$

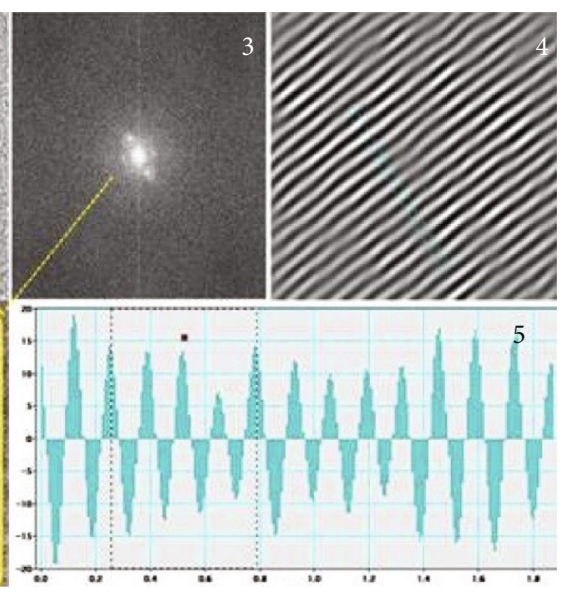

Figure 8: (a) TEM image of PVA-BMO1, (b) HRTEM image, (c) SAED ring, and (d) the magnified (2), FFT (3), IFFT (4), and profile of IFFT (5) image of (1) for $\mathrm{ZnO}$.

The SAED pattern, especially on PVA-TMOP1, indicates the presence of porous regions on the samples. The presence of several bright diffraction spots on the rings, especially for PVA-BMO1 and PVA-BMO2, confirms the crystallinity of the $\mathrm{ZnO}$, whereas the spots present out of the ring most probably is due to the $\alpha-\mathrm{Fe}_{2} \mathrm{O}_{3}$ or/and $\alpha-\mathrm{Mn}_{2} \mathrm{O}_{3}$ [54].

3.7. CV and EIS Studies. The electrochemical property of $\mathrm{ZnO}$, PVA-BMO1, PVA-BMO2, and PVA-TMOP1 was characterized by EIS and CV analytical techniques. The CV plots of these materials at different scan rates are given in Figure S10-12(a) and (b). With increasing the scan rate, the oxidation peak shifts towards more positive potential and a slight increment in redox peak current was observed. As reported [4], the presence of $\mathrm{Zn}(\mathrm{OH})_{4}^{2-}$ reduction peaks at a lower frequency for $\mathrm{ZnO}$ indicates the formation of photocorrosion. Its absence on the composites confirms the prevention of photocorrosion due to the enhancement of quantum efficiency and stability of the photocatalyst. For electrochemical improvement contrast, the $\mathrm{CV}$ plot of $\mathrm{ZnO}$ with the two binary and ternary nanocomposites at a scan rate of $30 \mathrm{mV} \mathrm{S}^{-1}$ is depicted in Figure S10-12(c). At the upper potential, the PVA-BMO1, PVA-BMO2, and PVATMOP1 CV curves show a greater current rise over the $\mathrm{ZnO}$ curve. This enhanced current response for the composites indicates the presence of an improved electron transport between the electrolyte medium and the electrode of the composite [55]. The electron transport potential order was determined to be PVA-TMOP1 > PVA-BMO1 > $\mathrm{PVA}-\mathrm{BMO} 2>\mathrm{ZnO}$. This obvious electron transport potential result for the composite is due to the formation of heterojunction between metal oxides. As confirmed on the SEM images, this may also be due to the porous nature of the nanocomposites.

The linear relationship plotted between the oxide peak current $\left(I_{p}\right)$ versus the scan rate $(v)$ is drawn as seen in Figure S10-12(d). The well-fitting of the linear plots for PVA-BMO1 and PVA-TMOP1 shows the adsorption controlled electrocatalytic process. Nonfitting of the PVA$\mathrm{BMO} 2$ linear plot indicates the diffusion-dependent 


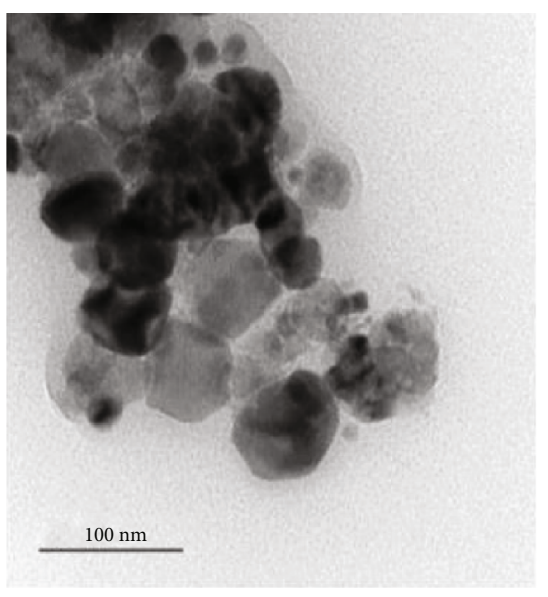

(a)

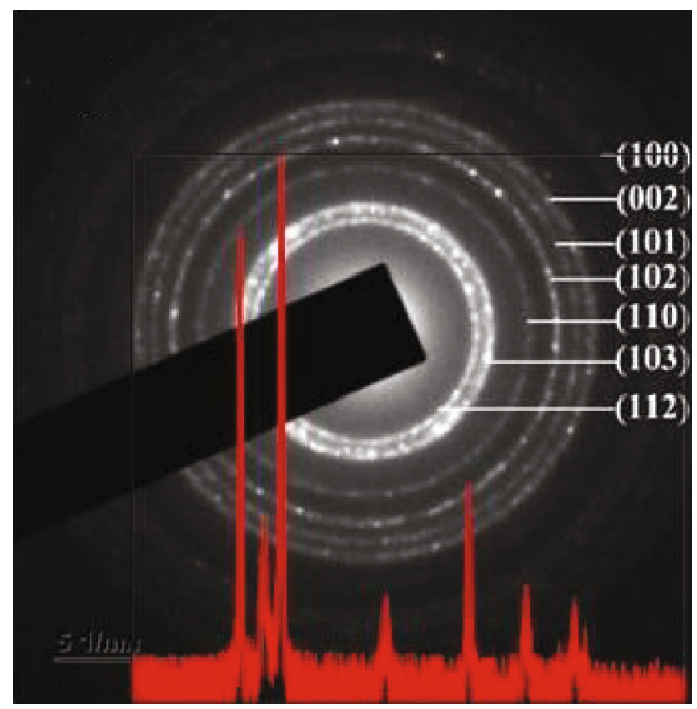

(c)

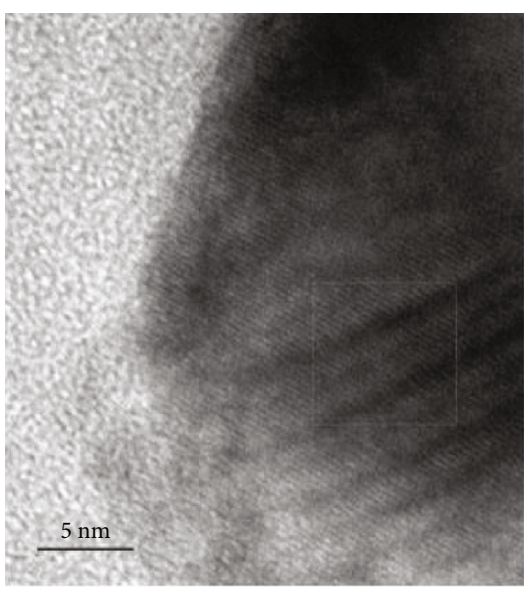

(b)
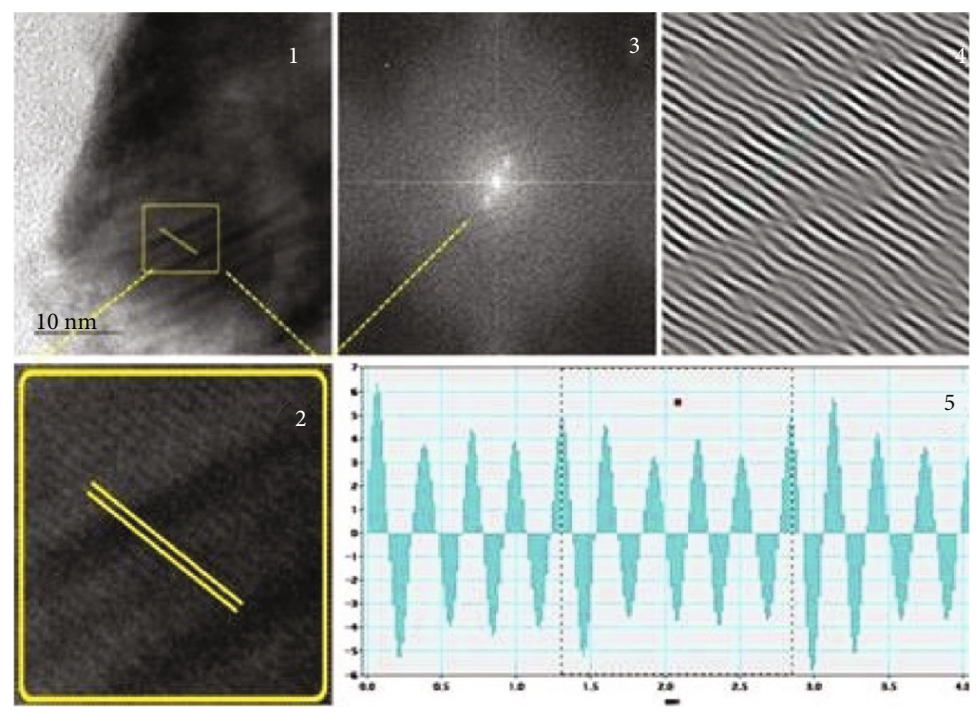

(d)

FIgURE 9: (a) TEM image of PVA-BMO2, (b) HRTEM image, (c) SAED ring, and (d) the magnified (2), FFT (3), IFFT (4), and profile of IFFT (5) image of (1) for $\mathrm{Mn}_{2} \mathrm{O}_{3}$.

electrocatalytic process. The combined $\mathrm{CV}$ plots of $\mathrm{ZnO}$, PVA-BMO1, PVA-BMO2, and PVA-TMOP1 are given in Figure 10(a). Compared to the other, the PVA-TMOP1 CV curve shows a greater electrochemical oxidation process.

To further understand the electrochemical properties of ZnO, PVA-BMO1, PVA-BMO2, and PVA-TMOP1 materials, the EIS test was conducted. As seen in Figure S1012(e) and Figure 10(b), the Nyquist plot was used to understand the resistance properties of the material. The semicircular portion at a higher frequency is equal to the electron transfer resistance $\left(R_{\mathrm{ct}}\right)$ at the contact interface of the electrode and electrolyte solution [56]. As seen in the combined EIS plots in Figure 10(b), the semicircular diameter of PVA-TMOP1 is much smaller than the other. This indicates that the PVA-TMOP1 has a higher electron transfer capability.

The value of $R_{\mathrm{ct}}$ is equal to the diameter of the semicircle [57]. The approximate $R_{\mathrm{ct}}$ value of $\mathrm{ZnO}, \mathrm{PVA}-\mathrm{BMO} 1, \mathrm{PVA}-$ $\mathrm{BMO} 2$, and PVA-TMOP1 was determined to be $65,25,61$, and $7 \Omega$, respectively. The result shows that the charge transfer capability is in the order of PVA-TMOP1>PVA$\mathrm{BMO} 1>\mathrm{PVA}-\mathrm{BMO} 2>\mathrm{ZnO}$. As confirmed on the TEM image, the increased conductivity of the composites may be due to the formation of heterojunction between the metal oxides and the porous nature of the materials. In advance, increasing the heterojunction to the ternary PVA-TMOP1 is showing enhanced charge transfer capability. As reported [58], this is most probably due to the more negative band position values of the composites compared to $\mathrm{ZnO}$. The linear portion at lower frequencies is known as the Warburg resistance, which is related to ion transport/diffusion from the electrolyte to the surfaces of the electrode.

\section{Conclusion}

Developing an efficient technique is the current necessity for the environmental waste management system. The novel sol-gel-self-propagation technique was developed for 


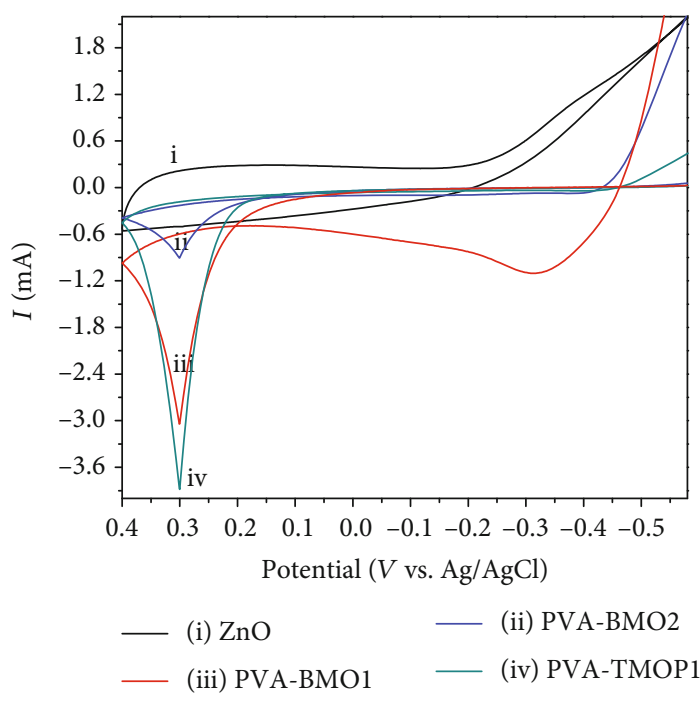

(a)

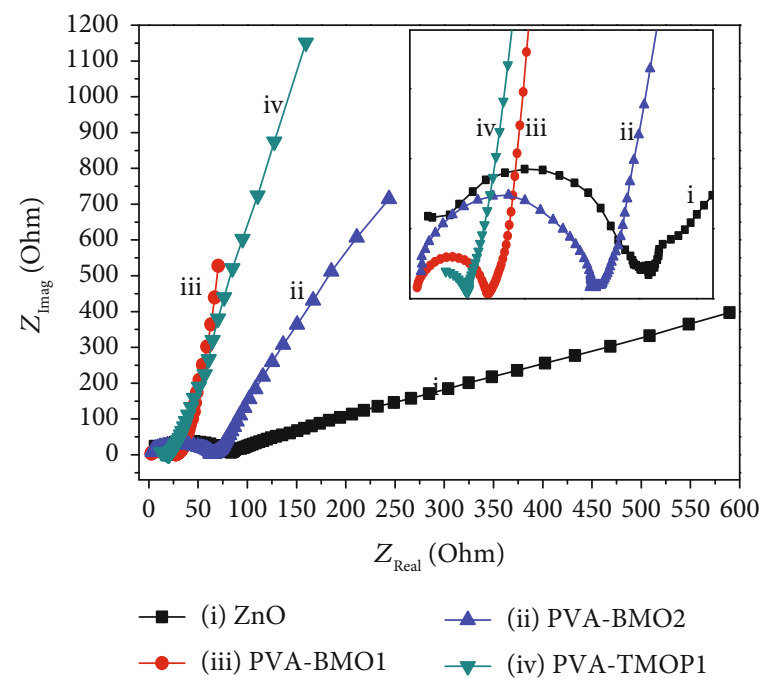

(b)

Figure 10: (a) CV plots of ZnO (i), PVA-BMO1 (iii), PVA-BMO2 (ii), and PVA-TMOP1 (iv); (b) the respective EIS spectra (inset: their magnified EIS plots).

the synthesis of porous materials. Compared to the coprecipitation, the sol-gel technique gave a better surface area to volume ratio result. The zinc nitrate precursor is giving good self-propagation progression than the zinc acetate salt precursor. The porous nature of the material was confirmed by SEM and BET analysis; in advance, this is confirmed from the SAED ring. Using the XRD pattern and TEM analysis, the particle size of the synthesized materials was confirmed to be in the nanorange $(\sim 7-70 \mathrm{~nm})$. The EDX and HRTEM analyses were used for the compositional investigation of the nanomaterials. The improvements in charge transfer synergy from single-binary-ternary were verified through $\mathrm{CV}$ and EIS techniques. The result shows the charge transfer capability to be in the order of ternar$y>$ binary $>$ single. The efficiency of these porous materials will also be tested for the photocatalytic degradation of dye.

\section{Data Availability}

The data used to support the findings of this study are included in the article and as supplementary material.

\section{Conflicts of Interest}

There are no conflicts to declare.

\section{Authors' Contributions}

The nanomaterial synthesis, characterization, and first draft of manuscript writing were performed by Buzuayehu Abebe. Sample characterization, advising, and manuscript writing improvement were performed by H C Ananda Murthy. Further, advising and manuscript writing improvement were performed by Enyew Amare Zereffa.

\section{Acknowledgments}

The authors are grateful to the management of Adama Science and Technology University for the financial support and Mr. Guta Amanu for his assistance during laboratory work.

\section{Supplementary Materials}

(i) The common analytical grade reagents used; (ii) characterization technique detail; (iii) figures that show the synthesis procedure, additional XRD, UV-Vis, FT-IR, BET, SEM, $\mathrm{CV}$, and EIS figures; and (iv) the XRD and BET data tables. (Supplementary Materials)

\section{References}

[1] M. Akkari, P. Aranda, C. Belver, J. Bedia, A. Ben Haj Amara, and E. Ruiz-Hitzky, " $\mathrm{ZnO} /$ sepiolite heterostructured materials for solar photocatalytic degradation of pharmaceuticals in wastewater," Applied Clay Science, vol. 156, pp. 104-109, 2018.

[2] C. B. Ong, L. Y. Ng, and A. W. Mohammad, "A review of $\mathrm{ZnO}$ nanoparticles as solar photocatalysts: synthesis, mechanisms and applications," Renewable and Sustainable Energy Reviews, vol. 81, pp. 536-551, 2018.

[3] B. Abebe, H. A. Murthy, and E. Amare, "Enhancing the photocatalytic efficiency of $\mathrm{ZnO}$ : defects, heterojunction, and optimization," Environmental Nanotechnology, Monitoring \& Management, vol. 14, article 100336, 2020.

[4] M. T. Qamar, M. Aslam, I. M. I. Ismail, N. Salah, and A. Hameed, "The assessment of the photocatalytic activity of magnetically retrievable $\mathrm{ZnO}$ coated $\gamma-\mathrm{Fe}_{2} \mathrm{O}_{3}$ in sunlight exposure," Chemical Engineering Journal, vol. 283, pp. 656-667, 2016.

[5] C. Gomez-Solís, J. C. Ballesteros, L. M. Torres-Martínez et al., "Rapid synthesis of $\mathrm{ZnO}$ nano-corncobs from Nital solution and its application in the photodegradation of methyl orange," 
Journal of Photochemistry and Photobiology A: Chemistry, vol. 298, pp. 49-54, 2015.

[6] M. Pirhashemi, A. Habibi-Yangjeh, and S. Rahim Pouran, "Review on the criteria anticipated for the fabrication of highly efficient $\mathrm{ZnO}$-based visible-light-driven photocatalysts," Journal of Industrial and Engineering Chemistry, vol. 62, pp. 1-25, 2018.

[7] M. Dehghani-Dashtabi, H. Hekmatara, and J. Seyed-Yazdi, "Synthesis and improved photoactivity of magnetic quaternary nanocomposites consisting of $\mathrm{Fe}_{3} \mathrm{O}_{4} @ \mathrm{ZnO}$ core@shell nanoparticles decorated on graphene-oxide grafted poly-citric acid," Physica B: Condensed Matter, vol. 553, pp. 11-17, 2019.

[8] L. Bigiani, C. Maccato, A. Gasparotto, C. Sada, and D. Barreca, "Structure and properties of $\mathrm{Mn}_{3} \mathrm{O}_{4}$ thin films grown on single crystal substrates by chemical vapor deposition," Materials Chemistry and Physics, vol. 223, pp. 591-596, 2019.

[9] A. L. Rosa, L. L. Tacca, T. Frauenheim, and E. N. Lima, "Electronic and optical properties of $\mathrm{Mn}$ impurities in ultra-thin $\mathrm{ZnO}$ nanowires: insights from density-functional theory," Physica E: Low-dimensional Systems and Nanostructures, vol. 109, pp. 6-10, 2019.

[10] X. Wang, Q. Li, C. Zhou, Z. Cao, and R. Zhang, "ZnO rod/ reduced graphene oxide sensitized by $\alpha-\mathrm{Fe}_{2} \mathrm{O}_{3}$ nanoparticles for effective visible-light photoreduction of $\mathrm{CO}_{2}$," Journal of Colloid and Interface Science, vol. 554, pp. 335-343, 2019.

[11] A. Jiamprasertboon, A. Kafizas, M. Sachs et al., "Heterojunction $\alpha-\mathrm{Fe}_{2} \mathrm{O}_{3} / \mathrm{ZnO}$ films with enhanced photocatalytic properties grown by aerosol-assisted chemical vapour deposition," Chemistry - A European Journal, vol. 25, no. 48, pp. 1133711345, 2019.

[12] S. Lee and H. Xu, "Size-dependent phase map and phase transformation kinetics for nanometric iron(III) oxides $(\gamma \rightarrow \varepsilon \rightarrow \alpha$ pathway)," Journal of Physical Chemistry C, vol. 120, no. 24, pp. 13316-13322, 2016.

[13] G. Yang, W. Yan, J. Wang, and H. Yang, "Fabrication and formation mechanism of $\mathrm{Mn}_{2} \mathrm{O}_{3}$ hollow nanofibers by singlespinneret electrospinning," CrystEngComm, vol. 16, no. 30, pp. 6907-6913, 2014.

[14] E. Moharreri, W. A. Hines, S. Biswas et al., "Comprehensive magnetic study of nanostructured mesoporous manganese oxide materials and implications for catalytic behavior," Chemistry of Materials, vol. 30, no. 3, pp. 1164-1177, 2018.

[15] J. Zhao, J. Nan, Z. Zhao, and N. Li, "Facile fabrication of novel $\mathrm{Mn}_{2} \mathrm{O}_{3}$ nanocubes with superior light-harvesting for ciprofloxacin degradation," Catalysis Communications, vol. 102, pp. 5-8, 2017.

[16] B. Abebe, H. C. A. Murthy, E. Zerefa, and Y. Adimasu, "PVA assisted $\mathrm{ZnO}$ based mesoporous ternary metal oxides nanomaterials: synthesis, optimization, and evaluation of antibacterial activity," Materials Research Express, vol. 7, no. 4, article 045011, 2020.

[17] H. Köse, Ş. Karaal, A. O. Aydın, and H. Akbulut, “A facile synthesis of zinc oxide/multiwalled carbon nanotube nanocomposite lithium ion battery anodes by sol-gel method," Journal of Power Sources, vol. 295, pp. 235-245, 2015.

[18] H. Wang, P. Zhou, R. Guo, Y. Wang, H. Zhan, and Y. Yuan, "Synthesis of rectorite $/ \mathrm{Fe}_{3} \mathrm{O}_{4} / \mathrm{ZnO}$ composites and their application for the removal of methylene blue dye," Catalysts, vol. 8 , no. 3, p. 107, 2018.

[19] C. M. Alder, J. D. Hayler, R. K. Henderson et al., "Updating and further expanding GSK's solvent sustainability guide," Green Chemistry, vol. 18, no. 13, pp. 3879-3890, 2016.
[20] L. Kumar Jangir, Y. Kumari, A. Kumar, M. Kumar, and K. Awasthi, "Investigation of luminescence and structural properties of $\mathrm{ZnO}$ nanoparticles, synthesized with different precursors," Materials Chemistry Frontiers, vol. 1, no. 7, pp. 1413-1421, 2017.

[21] Z. Wu and W. Wu, "Shape control of inorganic nanoparticles from solution," Nanoscale, vol. 8, pp. 1237-1259, 2016.

[22] M. Madkour, A. Bumajdad, and F. Al-sagheer, "To what extent do polymeric stabilizers affect nanoparticles characteristics?," Advances in Colloid and Interface Science, vol. 270, pp. 3853, 2019.

[23] S. Kumar, B. Krishnakumar, A. J. F. N. Sobral, and J. Koh, "Bio-based (chitosan/PVA/ZnO) nanocomposites film: thermally stable and photoluminescence material for removal of organic dye," Carbohydrate Polymers, vol. 205, pp. 559-564, 2019.

[24] A. V. Radhamani, K. M. Shareef, and M. S. R. Rao, " $\mathrm{ZnO} @ \mathrm{MnO}_{2}$ core-shell nanofiber cathodes for high performance asymmetric supercapacitors," ACS Applied Materials \& Interfaces, vol. 8, no. 44, pp. 30531-30542, 2016.

[25] B. Liu, Y. You, H. Zhang, H. Wu, J. Jin, and H. Liu, "Synthesis of $\mathrm{ZnO}$ nano-powders via a novel PVA-assisted freeze-drying process," RSC Advances, vol. 6, no. 111, pp. 110349-110355, 2016.

[26] B. Abebe, H. C. Ananda Murthy, E. Zerefa, and E. Abdisa, "Porous PVA/Zn-Fe-Mn oxide nanocomposites: methylene blue dye adsorption studies," Materials Research Express, vol. 7, no. 6, article 065002, 2020.

[27] H. Lachheb, F. Ajala, A. Hamrouni, A. Houas, F. Parrino, and L. Palmisano, "Electron transfer in $\mathrm{ZnO}-\mathrm{Fe}_{2} \mathrm{O}_{3}$ aqueous slurry systems and its effects on visible light photocatalytic activity," Catalysis Science \& Technology, vol. 7, no. 18, pp. 4041-4047, 2017.

[28] M. Xu, Q. Li, and H. Fan, "Monodisperse nanostructured $\mathrm{Fe}_{3} \mathrm{O}_{4} / \mathrm{ZnO}$ microrods using for waste water treatment," Advanced Powder Technology, vol. 25, no. 6, pp. 1715-1720, 2014.

[29] W. Yu, T. Liu, S. Cao, C. Wang, and C. Chen, "Constructing $\mathrm{MnO}_{2}$ /single crystalline $\mathrm{ZnO}$ nanorod hybrids with enhanced photocatalytic and antibacterial activity," Journal of Solid State Chemistry, vol. 239, pp. 131-138, 2016.

[30] M. F. Nsib, N. Naffati, A. Rayes, N. Moussa, and A. Houas, "Effect of some operational parameters on the hydrogen generation efficiency of $\mathrm{Ni}-\mathrm{ZnO} / \mathrm{PANI}$ composite under visible-light irradiation," Materials Research Bulletin, vol. 70, pp. 530-538, 2015.

[31] M. L. Maya-Treviño, M. Villanueva-Rodríguez, J. L. GuzmánMar, L. Hinojosa-Reyes, and A. Hernández-Ramírez, "Comparison of the solar photocatalytic activity of $\mathrm{ZnO}-\mathrm{Fe}_{2} \mathrm{O}_{3}$ and $\mathrm{ZnO}-\mathrm{Fe}^{0}$ on 2,4-D degradation in a CPC reactor," Photochemical \& Photobiological Sciences, vol. 14, no. 3, pp. 543549, 2015.

[32] A. H. Shah, E. Manikandan, M. Basheer Ahamed, D. Ahmad Mir, and S. Ahmad Mir, "Antibacterial and blue shift investigations in sol-gel synthesized $\mathrm{Cr}_{x} \mathrm{Zn}_{1-x} \mathrm{O}$ nanostructures," Journal of Luminescence, vol. 145, pp. 944-950, 2014.

[33] Z. Yang, X. Zong, Z. Ye, B. Zhao, Q. Wang, and P. Wang, "The application of complex multiple forklike $\mathrm{ZnO}$ nanostructures to rapid and ultrahigh sensitive hydrogen peroxide biosensors," Biomaterials, vol. 31, no. 29, pp. 7534-7541, 2010. 
[34] M. O. Fatehah, H. A. Aziz, and S. Stoll, "Stability of ZnO nanoparticles in solution. Influence of $\mathrm{pH}$, dissolution, aggregation and disaggregation effects," Journal of Colloid Science and Biotechnology, vol. 3, no. 1, pp. 75-84, 2014.

[35] F. A. Sigoli, M. R. Davolos, and M. Jafelicci, "Morphological evolution of zinc oxide originating from zinc hydroxide carbonate," Journal of Alloys and Compounds, vol. 262-263, pp. 292-295, 1997.

[36] I. S. Elashmawi and A. A. Menazea, “Different time's Nd:YAG laser-irradiated PVA/Ag nanocomposites: structural, optical, and electrical characterization," Journal of Materials Research and Technology, vol. 8, no. 2, pp. 1944-1951, 2019.

[37] I. S. Elashmawi, E. M. Abdelrazek, A. M. Hezma, and A. Rajeh, "Modification and development of electrical and magnetic properties of PVA/PEO incorporated with $\mathrm{MnCl}_{2}$," Physica B: Condensed Matter, vol. 434, no. 1, pp. 57-63, 2014.

[38] M.-S. Balogun, Z. Wu, Y. Luo et al., "High power density nitridated hematite $\left(\alpha-\mathrm{Fe}_{2} \mathrm{O}_{3}\right)$ nanorods as anode for highperformance flexible lithium ion batteries," Journal of Power Sources, vol. 308, pp. 7-17, 2016.

[39] C.-Y. Huang, K.-H. Hu, and Z.-H. Wei, "Comparison of cell behavior on pva/pva-gelatin electrospun nanofibers with random and aligned configuration," Scientific Reports, vol. 6, no. 1, article 37960, 2016.

[40] S.-Y. Lin, W.-T. Cheng, Y.-S. Wei, and H.-L. Lin, "DSC-FTIR microspectroscopy used to investigate the heat-induced intramolecular cyclic anhydride formation between Eudragit E and PVA copolymer," Polymer Journal, vol. 43, no. 6, pp. 577-580, 2011.

[41] R. Y. Hong, J. H. Li, L. L. Chen et al., "Synthesis, surface modification and photocatalytic property of $\mathrm{ZnO}$ nanoparticles," Powder Technology, vol. 189, no. 3, pp. 426-432, 2009.

[42] N. Rosman, W. N. W. Salleh, F. Aziz et al., "Electrospun nanofibers embedding $\mathrm{ZnO} / \mathrm{Ag}_{2} \mathrm{CO}_{3 /} \mathrm{Ag}_{2} \mathrm{O}$ heterojunction photocatalyst with enhanced photocatalytic activity," Catalysts, vol. 9, no. 7, p. 565, 2019.

[43] M. Thommes, K. Kaneko, A. V. Neimark et al., "Physisorption of gases, with special reference to the evaluation of surface area and pore size distribution (IUPAC technical report)," Pure and Applied Chemistry, vol. 87, no. 9-10, pp. 1051-1069, 2015.

[44] P. Kumar, K.-H. Kim, E. E. Kwon, and J. E. Szulejko, "Metalorganic frameworks for the control and management of air quality: advances and future direction," Journal of Materials Chemistry A, vol. 4, no. 2, pp. 345-361, 2016.

[45] J. Liu, J. He, L. Wang et al., "NiO-PTA supported on ZIF-8 as a highly effective catalyst for hydrocracking of jatropha oil," Scientific Reports, vol. 6, no. 1, article 23667, 2016.

[46] Z. ALOthman, "A review: fundamental aspects of silicate mesoporous materials," Materials, vol. 5, no. 12, pp. 28742902, 2012.

[47] X. S. Zhao, G. Q. Lu, and G. J. Millar, "Advances in mesoporous molecular sieve MCM-41," Industrial and Engineering Chemistry Research, vol. 35, no. 7, pp. 2075-2090, 1996.

[48] B. Abebe and H. C. Ananda Murthy, "Synthesis and characterization of Ti-Fe oxide nanomaterials for lead removal," Journal of Nanomaterials, vol. 2018, 10 pages, 2018.

[49] B. Abebe, H. C. Ananda Murthy, and Y. Dessie, "Synthesis and characterization of $\mathrm{Ti}-\mathrm{Fe}$ oxide nanomaterials: adsorptiondegradation of methyl orange dye," Arabian Journal for Science and Engineering, vol. 45, no. 6, pp. 4609-4620, 2020.

[50] Z. Zeng, W. Zhang, D. M. Arvapalli et al., "A fluorescenceelectrochemical study of carbon nanodots (CNDs) in bio- and photoelectronic applications and energy gap investigation," Physical Chemistry Chemical Physics, vol. 19, no. 30, pp. 20101-20109, 2017.

[51] D. Muñoz-Rojas, J. Oró-Solé, and P. Gómez-Romero, "From nanosnakes to nanosheets: a matrix-mediated shape evolution," Journal of Physical Chemistry C, vol. 112, no. 51, pp. 20312-20318, 2008.

[52] X. Liu, L. Ye, S. Liu, Y. Li, and X. Ji, "Photocatalytic reduction of $\mathrm{CO}_{2}$ by $\mathrm{ZnO}$ micro/nanomaterials with different morphologies and ratios of $\{0001\}$ facets," Scientific Reports, vol. 6, no. 1 , article $38474,2016$.

[53] J. Zhao, Z. Zhao, N. Li, J. Nan, R. Yu, and J. Du, "Visible-lightdriven photocatalytic degradation of ciprofloxacin by a ternary $\mathrm{Mn}_{2} \mathrm{O}_{3} / \mathrm{Mn}_{3} \mathrm{O}_{4} / \mathrm{MnO}_{2}$ valence state heterojunction," Chemical Engineering Journal, vol. 353, pp. 805-813, 2018.

[54] T. Zhai, S. Xie, Y. Zhao et al., "Controllable synthesis of hierarchical ZnO nanodisks for highly photocatalytic activity," CrystEngComm, vol. 14, no. 5, pp. 1850-1855, 2012.

[55] E. Gopinathan, G. Viruthagiri, N. Shanmugam, and S. Sathiyapriya, "Optical, surface analysis and antibacterial activity of $\mathrm{ZnO}-\mathrm{CuO}$ doped cerium oxide nanoparticles," Optik, vol. 126, no. 24, pp. 5830-5835, 2015.

[56] Z. Li, Y. Mi, X. Liu, S. Liu, S. Yang, and J. Wang, "Flexible graphene/ $\mathrm{MnO}_{2}$ composite papers for supercapacitor electrodes," Journal of Materials Chemistry, vol. 21, no. 38, article 14706, 2011.

[57] Z. Li, Z. Zhou, G. Yun, K. Shi, X. Lv, and B. Yang, "High-performance solid-state supercapacitors based on graphene- $\mathrm{ZnO}$ hybrid nanocomposites," Nanoscale Research Letters, vol. 8, no. 1, p. 473, 2013.

[58] R. D. Suryavanshi and K. Y. Rajpure, "Spray deposited $\mathrm{Fe}_{2} \mathrm{O}_{3}$ and stratified $\mathrm{Fe}_{2} \mathrm{O}_{3} / \mathrm{ZnO}$ novel photoelectrode for photoelectrocatalytic degradation of benzoic acid under solar light illumination," Journal of Photochemistry and Photobiology A: Chemistry, vol. 357, pp. 72-80, 2018. 